\title{
Understanding the destabilizing role for surface tension in planar shear flows in terms of wave interaction
}

\author{
L. Biancofiore, ${ }^{1}$ E. Heifetz, ${ }^{2}$ J. Hoepffner, ${ }^{3}$ and F. Gallaire ${ }^{4}$ \\ ${ }^{1}$ Department of Mechanical Engineering, Bilkent University, 06800 Bilkent, Ankara, Turkey \\ ${ }^{2}$ Department of Geophysics, School of Earth Sciences, Tel Aviv University, Tel Aviv, 69978, Israel \\ ${ }^{3}$ CNRS (UMR 7190), Université Pierre et Marie Curie, Institut Jean le Rond d'Alembert, 75005, Paris, France \\ ${ }^{4}$ EPFL/LFMI, Route Cantonale, Lausanne, CH-1015, Switzerland
}

(Received 1 February 2017; published 4 October 2017)

\begin{abstract}
Both surface tension and buoyancy force in stable stratification act to restore perturbed interfaces back to their initial positions. Hence, both are intuitively considered as stabilizing agents. Nevertheless, the Taylor-Caulfield instability is a counterexample in which the presence of buoyancy forces in stable stratification destabilize shear flows. An explanation for this instability lies in the fact that stable stratification supports the existence of gravity waves. When two vertically separated gravity waves propagate horizontally against the shear, they may become phase locked and amplify each other to form a resonance instability. Surface tension is similar to buoyancy but its restoring mechanism is more efficient at small wavelengths. Here, we show how a modification of the Taylor-Caulfield configuration, including two interfaces between three stably stratified immiscible fluids, supports interfacial capillary gravity whose interaction yields resonance instability. Furthermore, when the three fluids have the same density, an instability arises solely due to a pure counterpropagating capillary wave resonance. The linear stability analysis predicts a maximum growth rate of the pure capillary wave instability for an intermediate value of surface tension corresponding to $\mathrm{We}^{-1}=5$, where We denotes the Weber number. We perform direct numerical nonlinear simulation of this flow and find nonlinear destabilization when $2 \leqslant \mathrm{We}^{-1} \leqslant 10$, in good agreement with the linear stability analysis. The instability is present also when viscosity is introduced, although it is gradually damped and eventually quenched.
\end{abstract}

DOI: 10.1103/PhysRevFluids.2.103901

\section{INTRODUCTION}

Surface tension acts as a restoring force in shear flows. Perturbed interfaces tend to go back to their initial position under its restoring influence. For this reason, surface tension is often considered intuitively as a stabilizing force in plane shear flows. For instance, the effect of the surface tension at the interface of two immiscible fluids damps the Kelvin-Helmholtz instability [1,2]. In axisymmetric shear flows, however, the same reasoning does not hold true, and surface tension can become destabilizing as in the Rayleigh-Plateau instability [3,4] of a liquid jet.

A destabilizing influence of surface tension was recently found in planar jets and wakes [5-7] by means of both linear global analysis and direct numerical simulations (DNS). This counterintuitive destabilization is caused by the appearance of a second unstable mode acting particularly at large wave numbers. The presence of this second unstable mode was explained by Biancofiore et al. [8] using the kernel wave (KW) perspective. This perspective was first developed in atmospheric science to explain the baroclinic instability mechanism for cyclone genesis resulting from interaction in a distance between Rossby waves [9]. The instability in flows featuring two distinct potential vorticity gradients can be interpreted in terms of the interaction between the two Rossby waves created at the vorticity edges. For a comprehensive review of the KW perspective, the reader is referred to Ref. [10]. The second mode destabilizing planar immiscible wakes can be interpreted as a Rossby-capillary instability [8], i.e., the interaction between the capillary waves at the interfaces with the Rossby waves created at the vorticity edges of the wake flow velocity profile. 
Another restoring force is buoyancy for stably stratified flows. Like surface tension, buoyancy under stable stratification has also a damping effect on the Kelvin-Helmotz instability [1]. However, the Holmboe instability [11] is a counterexample in which the presence of buoyancy forces in stable stratification destabilizes shear flows. In particular, a Holmboe mode is formed by the interaction between one Rossby and one gravity wave [12]. This is similar to what occurred for the Rossbycapillary instability in two immiscible fluid wakes [8]. An even simpler configuration where the buoyancy can destabilize a plane parallel flow is the Taylor-Caulfield instability [13,14]. This instability is due to interaction between two gravity waves created by stable stratification. The gravity waves may become phase locked and amplify each other in a distance to form a resonance instability $[15,16]$. Because of the similarity between gravity and capillary waves, it is natural to imagine that an instability equivalent to the Taylor-Caulfield instability can be obtained with the interaction of two capillary waves.

In order to highlight the role of the interacting capillary waves in shear flows, we analyze a two-dimensional stratified three-fluid flow, i.e., a Taylor-Caulfied configuration including surface tension. The work is structured as follows: In Sec. II, we present the analyzed model and the governing equations. In Sec. III, we show (i) how two capillary-gravity waves are generated at each of the two interfaces and (ii) how these waves can interact together to generate an instability, a Taylor-Caulfield instability modified by capillarity. The linear stability analysis of such a configuration is then discussed in detail in Sec. IV. The stratification is finally suppressed in Sec. V, demonstrating the possible existence of a pure capillary planar instability. This instability, generated by the interaction of two pure capillary waves, is further interpreted by means of the KW perspective and found in direct numerical simulations (DNS) of viscous flows. Conclusions are finally drawn in Sec. VI.

\section{MODEL AND LINEARIZED GOVERNING EQUATIONS}

\section{A. Model: Taylor-Caulfield setup with surface tension}

As seen in Fig. 1, the model considered in this study consists of a layer of fluid 2 sandwiched between two unconfined immiscible fluids 1 and 3 , all three submitted to the same constant uniform shear $U\left(y^{*}\right)=U_{y} y^{*}$, where $U_{y}$ is the constant shear rate. The three layers are assumed to be inviscid and are stratified with growing densities $\rho_{1}<\rho_{2}<\rho_{3}$. The base-dimensional density profile can be thus written as

$$
\rho\left(y^{*}\right)=\rho_{3}+\Delta \rho_{B} \mathcal{H}\left(y^{*}+h\right)+\Delta \rho_{T} \mathcal{H}\left(y^{*}-h\right),
$$

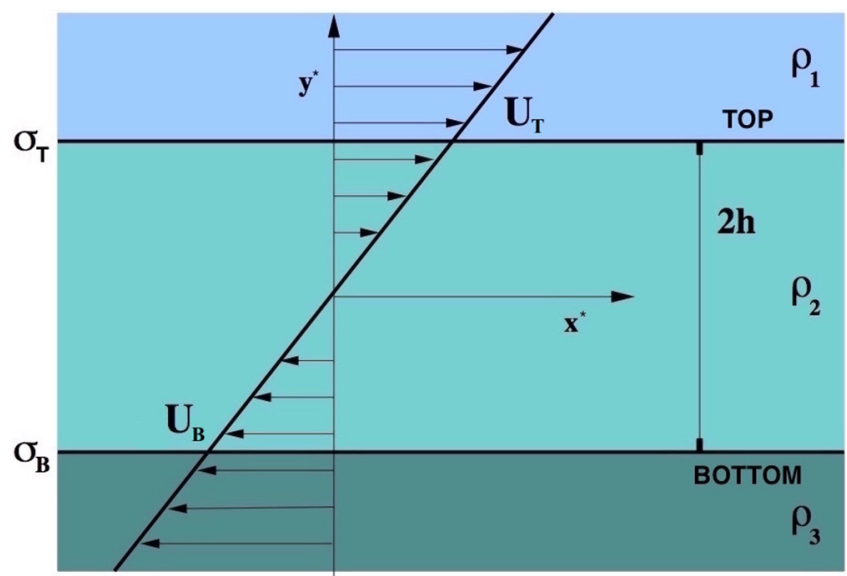

FIG. 1. The model flow. The base-dimensional velocity profile is $U\left(y^{*}\right)=U_{y} y^{*}$. 
where $\mathcal{H}$ is the Heaviside function, $\Delta \rho_{T}=\left(\rho_{1}-\rho_{2}\right)$, and $\Delta \rho_{B}=\left(\rho_{2}-\rho_{3}\right)$. At the two top $(T)$ and bottom $(B)$ interfaces, both buoyancy $b$ and interfacial tensions, $\sigma_{T}$ and $\sigma_{B}$, respectively, operate as restoring forces. The buoyancy gradient is $b_{y}=\Delta b_{T} \delta\left(y^{*}-h\right)+\Delta b_{B} \delta\left(y^{*}+h\right)$, where $\Delta b_{T, B}=$ $-\frac{\Delta \rho_{T, B}}{\rho_{3}} g$ and $g$ is the gravity. Note that the density of the lower fluid $\rho_{3}$ is chosen as a reference density. The distance between the two interfaces is $2 h$, while the velocity of the interfaces is $U_{T}=U_{y} h$ and $U_{B}=-U_{y} h$, respectively. This model can be seen as an extension of the Taylor-Caulfield flow $[13,14]$, where surface tension operates at the interfaces.

\section{B. Vorticity-displacement formulation}

The momentum and continuity equations linearized with respect to the velocity profile $U(y)$ are

$$
\begin{aligned}
\frac{D u^{*}}{D t^{*}} & =-v^{*} U_{y}-\frac{1}{\rho_{3}} \frac{\partial p^{*}}{\partial x^{*}}, \\
\frac{D v^{*}}{D t^{*}} & =b^{*}+\sigma_{T} C_{T}+\sigma_{B} C_{B}-\frac{1}{\rho_{3}} \frac{\partial p^{*}}{\partial y^{*}}, \\
C_{T} & =\kappa_{T}^{*} \delta\left(y^{*}-h\right) \text { and } C_{B}=\kappa_{B}^{*} \delta\left(y^{*}+h\right), \\
\frac{D b^{*}}{D t^{*}} & =-v^{*} b_{y}^{*}, \\
\frac{\partial u^{*}}{\partial x^{*}}+\frac{\partial v^{*}}{\partial y^{*}} & =0,
\end{aligned}
$$

where $\left(D / D t^{*}\right) \equiv\left(\partial / \partial t^{*}\right)+U^{*}\left(\partial / \partial x^{*}\right), \mathbf{u}^{*}=\left(u^{*}, v^{*}\right)$ is the perturbation velocity vector, $p^{*}$ is the perturbation pressure, $\sigma_{T / B}$ are the surface tensions, $C_{T / B}$ are the localized capillary forces at the interfaces $T$ and $B$, respectively, $\kappa_{T, B}^{*}=\frac{\partial^{2} \zeta_{T, B}^{*}}{\partial x^{* 2}}$ are the approximated interface curvatures, $\zeta_{T, B}^{*}$ are the perturbation cross-stream displacements, $\delta$ symbolizes the Dirac $\delta$ function, $b^{*}=\left(-\frac{\rho^{*}}{\rho_{3}}\right) g$ is the perturbation buoyancy, $b_{y}^{*}=-\frac{g}{\rho_{3}} \frac{d \rho^{*}}{d y^{*}}$ is the vertical buoyancy gradient, and $\rho^{*}$ is the perturbation density.

Equation set (2) can be transformed after some algebra to

$$
\begin{aligned}
\frac{D q^{*}}{D t^{*}} & =-\left(\Delta b_{T}+\sigma_{T}\right) \frac{\partial \zeta_{T}^{*}}{\partial x^{*}} \delta\left(y^{*}-h\right)-\left(\Delta b_{B}+\sigma_{B}\right) \frac{\partial \zeta_{B}^{*}}{\partial x^{*}} \delta\left(y^{*}-h\right) \\
\frac{D \zeta_{T, B}^{*}}{D t^{*}} & =v^{*}
\end{aligned}
$$

where $q^{*}$ is the perturbation vorticity, $q^{*}=\left(\partial v^{*} / \partial x^{*}\right)-\left(\partial u^{*} / \partial y^{*}\right)$.

We use $h$ and $1 / U_{y}$ as reference scales for the length and the time, respectively, and we indicate dimensionless quantities without an asterisk. Then for a single Fourier component with wave number $k$ of the form $e^{i k x}$, Eq. (3) can be rewritten as

$$
\begin{aligned}
\left(\frac{\partial}{\partial t}+i k y\right) q & =-i k\left(\mathrm{Ri}_{T}+k^{2} \mathrm{We}_{T}^{-1}\right) \zeta_{T} \delta(y-1)-i k\left(\mathrm{Ri}_{B}+k^{2} \mathrm{We}_{B}^{-1}\right) \zeta_{B} \delta(y+1) \\
\left(\frac{\partial}{\partial t}+i k y\right) \zeta_{T, B} & =\int_{y^{\prime}} q\left(y^{\prime}\right) G\left(y, y^{\prime}, k\right) d y^{\prime}
\end{aligned}
$$

where $\mathrm{Ri}_{T, B}=\frac{\Delta b_{T, B}}{h U_{y}^{2}}$ is the Richardson number and $\mathrm{We}_{T, B}=\frac{h^{3} \rho_{3} U_{y}^{2}}{\sigma_{T, B}}$ is the Weber number. Note that we introduced the Green's function

$$
G\left(y, y^{\prime}, k\right)=-\frac{i}{2} \exp \left(-k\left|y-y^{\prime}\right|\right)
$$

where $\nabla^{2} G\left(y, y^{\prime}, k\right)=-k^{2} G+G_{y y}=i k \delta\left(y-y^{\prime}\right)$ and $G$ vanishes as well for $y \rightarrow \pm \infty$ [17]. 
As usual in the kernel perspective theory $[8,16]$, we can see the vorticity perturbation decomposed in two vorticity waves localized in $y= \pm 1$. Thus, we can write, indicating with $T$ and $B$ the upper and lower interfaces, respectively,

$$
q=\left[\hat{q}_{T} \delta(y-1)+\hat{q}_{B} \delta(y+1)\right] .
$$

Therefore, we can write explicitly Eq. (4) for the two jumps:

$$
\begin{aligned}
& \left(\frac{\partial}{\partial t}+i k\right) \hat{q}_{T}=-i k\left(\mathrm{Ri}_{T}+k^{2} \mathrm{We}_{T}^{-1}\right) \zeta_{T}, \\
& \left(\frac{\partial}{\partial t}+i k\right) \zeta_{T}=-\frac{i}{2}\left[\hat{q}_{T}+\hat{q}_{B} \exp (-2 k)\right], \\
& \left(\frac{\partial}{\partial t}-i k\right) \hat{q}_{B}=-i k\left(\operatorname{Ri}_{B}+k^{2} \mathrm{We}_{B}^{-1}\right) \zeta_{B}, \\
& \left(\frac{\partial}{\partial t}-i k\right) \zeta_{B}=-\frac{i}{2}\left[\hat{q}_{T} \exp (-2 k)+\hat{q}_{B}\right] .
\end{aligned}
$$

Note that the dimensionless basic state velocity at the interfaces is \pm 1 . We can write the dynamical system depicted by Eq. (7) in the matrix form

$$
\begin{aligned}
\dot{\boldsymbol{\xi}} & =\boldsymbol{M} \boldsymbol{\xi}, \quad \boldsymbol{\xi}=\left[\begin{array}{l}
q_{T} \\
\zeta_{T} \\
q_{B} \\
\zeta_{B}
\end{array}\right], \quad \text { and } \\
\boldsymbol{M} & =\left[\begin{array}{cccc}
-i k & -i k^{3} W e_{T}^{-1}-i k R i_{T} & 0 & 0 \\
-\frac{i}{2} & -i k & -i R & 0 \\
0 & 0 & i k & -i k^{3} W e_{B}^{-1}-i k R i_{B} \\
-i R & 0 & -\frac{i}{2} & i k
\end{array}\right],
\end{aligned}
$$

where $R$ is the interaction coefficient between the two interfaces $R=\frac{e^{-2 k}}{2}$.

\section{PHYSICAL INTERPRETATION IN TERMS OF WAVE RESONANCE}

In this section, we describe the system in term of the kernel wave perspective. First, in Sec. III A, we illustrate the capillary-gravity wave dynamics in isolation, whereas their interaction in a distance is discussed in Sec. III B.

\section{A. Interfacial capillary-gravity waves}

Consider each interface in isolation, i.e., with the interaction coefficient $R=0$; then Eq. (7) is reduced to

$$
\begin{aligned}
& \left(\frac{\partial}{\partial t}+i k\right) \hat{q}_{T}=-i k F_{T}(k) \zeta_{T}, \\
& \left(\frac{\partial}{\partial t}+i k\right) \zeta_{T}=-\frac{i}{2} \hat{q}_{T}, \\
& \left(\frac{\partial}{\partial t}-i k\right) \hat{q}_{B}=-i k F_{B}(k) \zeta_{B}, \\
& \left(\frac{\partial}{\partial t}-i k\right) \zeta_{B}=-\frac{i}{2} \hat{q}_{B},
\end{aligned}
$$


where

$$
F_{T, B}(k)=R i_{T, B}+k^{2} W e_{T, B}^{-1}
$$

represents the dependency of the buoyancy-capillary restoring effects in the wave number $k$ at the top and bottom layers. The eigenvalues and eigenmodes of this uncoupled system are

$$
\begin{aligned}
& \omega_{T}^{ \pm}=k \pm \sqrt{\frac{F_{T} k}{2}}, \\
& \omega_{B}^{ \pm}=-k \pm \sqrt{\frac{F_{B} k}{2}}, \\
& \xi_{T}^{ \pm}=\left[1, \pm \sqrt{\frac{1}{2 k F_{T}}}, 0,0\right]^{t}, \\
& \xi_{B}^{ \pm}=\left[0,0,1, \pm \sqrt{\frac{1}{2 k F_{B}}}\right]^{t},
\end{aligned}
$$

where the $+(-)$ refers to the wave which propagates towards the positive (negative) $x$ direction relative to the velocity of the interface.

These eigenvalues correspond to a transport term $( \pm k)$ plus the pure neutral waves with propagation speed

$$
c_{T, B}=\sqrt{\frac{F_{T, B}}{2 k}}=\sqrt{\frac{\mathrm{Ri}_{T, B}}{2 k}+\frac{k \mathrm{We}_{T, B}^{-1}}{2}} .
$$

Hence, for pure deep water interfacial gravity waves (in the absence of surface tension), the intrinsic phase speed is increasing with the wavelength $\ell=\frac{2 \pi}{k}, c_{T, B}=\frac{1}{2} \sqrt{\frac{\mathrm{Ri}_{T, B} \ell}{\pi}}$, whereas for pure capillary waves (without the density stratification) $c_{T, B}$ decreases with the wavelength $c_{T, B}=\sqrt{\frac{\pi \mathrm{We}_{T, B}^{-1}}{\ell}}$. The interface deformation and the vorticity perturbation are either in or out of phase depending on the propagating direction relative to the interface velocity. In Fig. 2, the propagation mechanism
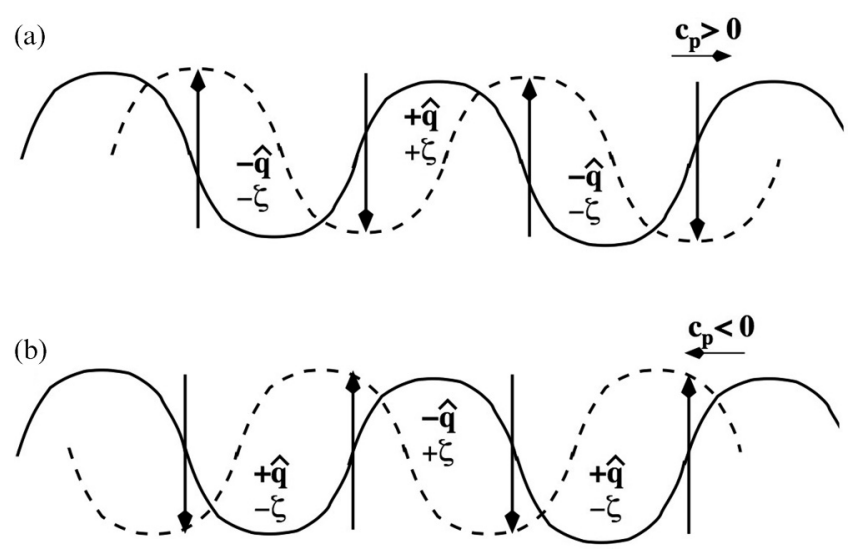

FIG. 2. The propagation mechanism for (a) positive $c_{p}$, i.e., the propagation speed relative to the interface velocity, with vorticity and displacement in phase, and (b) negative $c_{p}$ with vorticity and displacement in antiphase. Continuous lines represent the wave at time $t$, while the dashed lines show the wave at time $t+\delta t$. The arrows show the vertical velocity associated with the vorticity perturbation field. 
is illustrated: The vertical arrows represent the vertical velocity field associated with the vorticity perturbation so that upward (downward) motions are located a quarter of wavelength to the right (left) of positive vorticity anomalies. The restoring force (either by buoyancy, surface tension, or both) acts to generate positive (negative) vorticity anomalies where $\frac{\partial \zeta}{\partial x}$ is negative (positive) [16]; see also Eq. (3a). Hence, one can observe that the mechanism by which both the vorticity and the displacement propagate in concert is such that for positive (negative) $c_{p}, \hat{q}$ and $\zeta$ are in phase (antiphase) as indicated from Eqs. 11(c) and 11(d).

\section{B. Wave interaction}

Following the procedure in Ref. [15] but including the capillarity term, the dynamical system (8) can be rewritten in the basis of the uncoupled eigenmodes [defined in Eqs. 11(c) and 11(d)] using the transformation matrix $\mathbf{q}=\boldsymbol{T} \boldsymbol{\xi}$,

$$
\boldsymbol{T}=\left[\begin{array}{cccc}
1 & 1 & 0 & 0 \\
\sqrt{\frac{1}{2 k F_{T}}} & -\sqrt{\frac{1}{2 k F_{T}}} & 0 & 0 \\
0 & 0 & 1 & 1 \\
0 & 0 & \sqrt{\frac{1}{2 k F_{B}}} & -\sqrt{\frac{1}{2 k F_{B}}}
\end{array}\right],
$$

yielding

$$
\dot{\mathbf{q}}=\left(\boldsymbol{T}^{-1} \boldsymbol{M T}\right) \mathbf{q}=\bar{M} \mathbf{q},
$$

where

$$
\overline{\boldsymbol{M}}=i\left[\begin{array}{cccc}
-k-\sqrt{\frac{k F_{T}}{2}} & 0 & -R \sqrt{\frac{k F_{T}}{2}} & -R \sqrt{\frac{k F_{T}}{2}} \\
0 & -k+\sqrt{\frac{k F_{T}}{2}} & R \sqrt{\frac{k F_{T}}{2}} & R \sqrt{\frac{k F_{T}}{2}} \\
-R \sqrt{\frac{k F_{B}}{2}} & -R \sqrt{\frac{k F_{B}}{2}} & k-\sqrt{\frac{k F_{B}}{2}} & 0 \\
R \sqrt{\frac{k F_{B}}{2}} & R \sqrt{\frac{k F_{B}}{2}} & 0 & k+\sqrt{\frac{k F_{B}}{2}}
\end{array}\right] .
$$

The eigenvalues $\lambda=\lambda_{r}+i \lambda_{i}$ of the matrix $\bar{M}$ represent the normal modes of the systems. In particular, the real part of the eigenvalues $\lambda_{r}=k c_{i}$ is the growth rate of the instability while their imaginary part $\lambda_{i}=-k c_{r}$ is the frequency.

\section{LINEAR STABILITY ANALYSIS}

In this section, we discuss the linear stability analysis of the Taylor-Caulfield flow, including the capillary effects. In particular, the influence of the surface tension is discussed in Sec. IV A. Afterwards, the dynamics of the counterpropagation interaction is described in Sec. IV B before a final discussion on the normal mode instability mechanism (Sec. IV C). Hereafter, we consider the symmetric configuration $\mathrm{Ri}_{T}=\mathrm{Ri}_{B}=\mathrm{Ri}, \mathrm{We}_{T}=\mathrm{We}_{B}=\mathrm{We}$, so that $F_{T}=F_{B}=F$.

\section{A. The influence of surface tension}

In Figs. 3(a) and 3(b), the growth rate $\lambda_{r}$ and the phase speeds $c_{r}=-\frac{\lambda_{i}}{k}$ are plotted as a function of the wave number $k$ with varying Weber number for a fixed Richardson number $\mathrm{Ri}=0.5$. Both the growth rate and the cutoff wave number are first enhanced when the surface tension is introduced; however, as $\mathrm{We}^{-1}$ is further increased, they both get attenuated. Observe that at $\mathrm{We}^{-1}=1$, a separate finite bandwidth of large wave numbers allows instability as well. Due to the zero mean velocity of the considered velocity profile, unstable modes have $c_{r}=0$. In contrast, neutral modes are characterized by $c_{r} \neq 0$; they come into pairs with opposite phase velocities. 

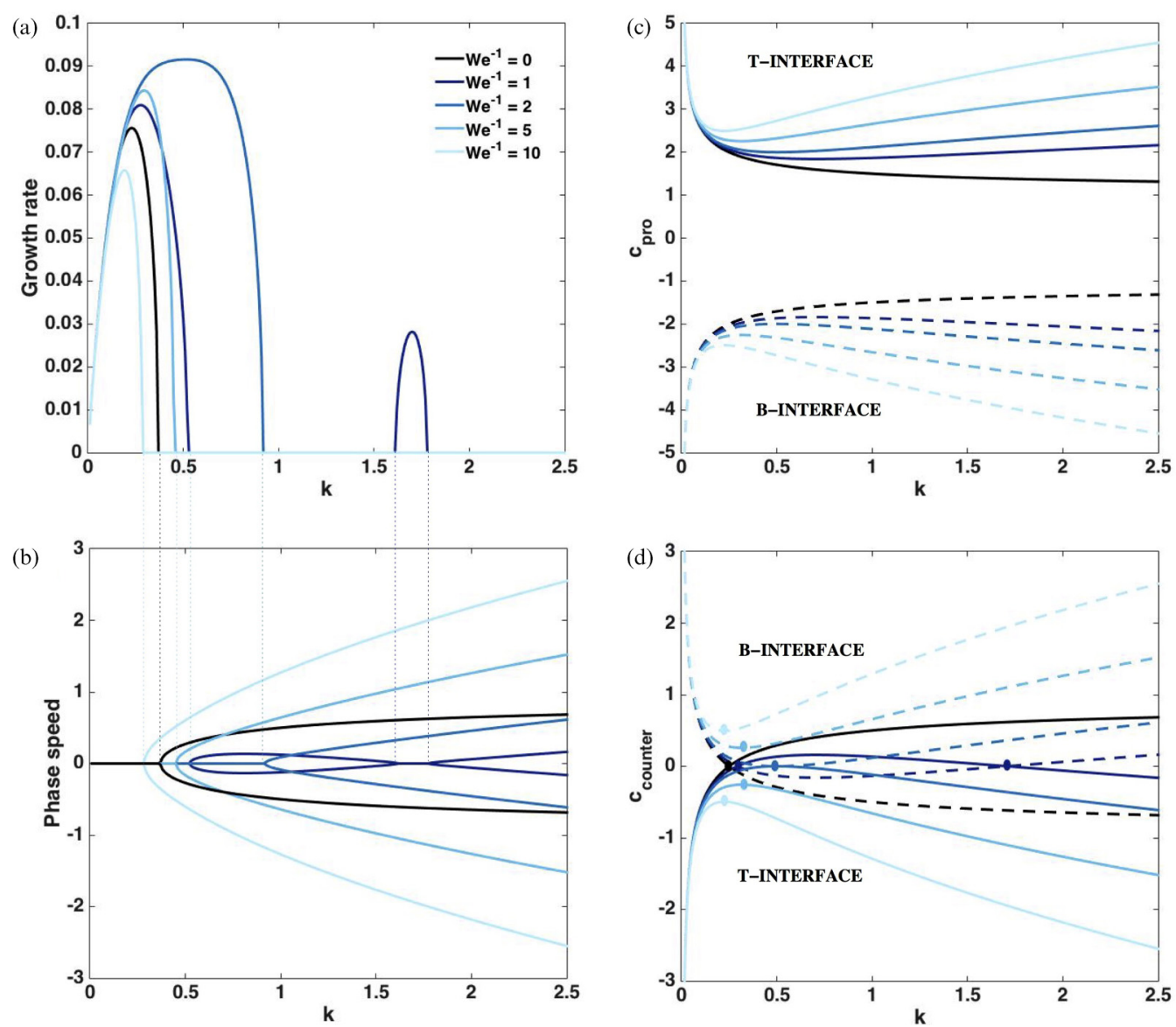

FIG. 3. (a) Growth rate and (b) phase speed of the normal modes as function of wave number for $\mathrm{Ri}=0.5$ and different values of $\mathrm{We}^{-1}$. Phase speed of the associated pro-propagating and counterpropagating waves in isolation are presented in panels (c) and (d), respectively. Continuous lines depict the waves at the upper interface $(T)$ and dashed lines at the lower interface $(B)$. The circles in panel $(d)$ represent the wave number for which the two waves are closer to each other.

In Fig. 3(c), we show the phase speeds of the pro-propagating waves $c_{T}^{+}$and $c_{B}^{-}$(further referred to as $c_{\text {pro }}$ ), taken in isolation, i.e., without considering the interaction. In contrast, the counterpropagating waves $c_{T}^{-}$and $c_{B}^{+}$, depicted in Fig. 3(d), are denoted as $c_{\text {counter }}$. The term pro-propagation (respectively counterpropagation) relates to the sign of the intrinsic phase velocity when compared to the velocity of the interface. The dependance of the phase speed with respect to the wave number $k$ is typical to capillary-gravity waves [18] with an extremum attained for an intermediate value of the wave number. It is striking to observe that modal instability appears when the counterpropagating waves $c_{T}^{-}$and $c_{B}^{+}$have comparable phase speeds (depicted by circles). The second instability branch in the finite wave-number range observed for $\mathrm{We}^{-1}=1$ is seen in particular to correspond to situations where $c_{T}^{-}=c_{B}^{+}$. This is also true for the extended cutoff wave number for $\mathrm{We}^{-1}=2$. In contrast, the pro-propagating waves at the two interfaces have velocities that are too different to enable a destabilizing interaction. These facts point out how phase-locked counterpropagating waves generate the instability. We will discuss this mechanism more closely in Sec. IV B.

The destabilizing influence of the surface tension can be observed clearly in Fig. 4 where the contours of positive growth rate are depicted in the plane $k-\mathrm{We}^{-1}$ for different values of the 

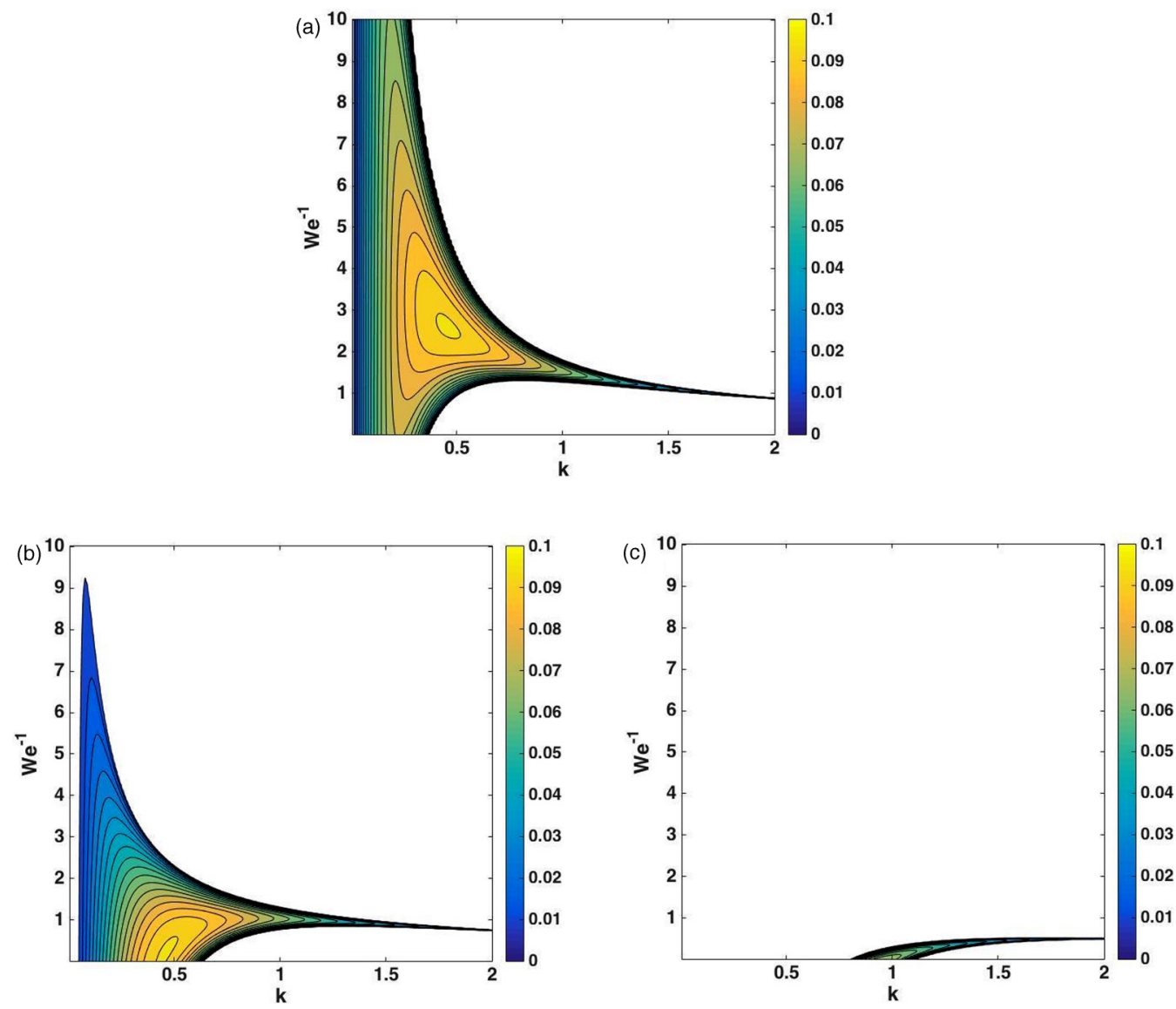

FIG. 4. Growth rate in the $k-\mathrm{We}^{-1}$ plane for (a) $\mathrm{Ri}=0.5$, (b) $\mathrm{Ri}=1$, and (c) $\mathrm{Ri}=2$.

Richardson number: (a) $\mathrm{Ri}=0.5$, (b) $\mathrm{Ri}=1$, and (c) $\mathrm{Ri}=2$. One can notice that the destabilization is stronger at weak stratification, while for $\mathrm{Ri}=2$ surface tension is only stabilizing. The location of the maximum of the instability depends on the degree of stratification. For $\mathrm{Ri}=0.5$, the maximum corresponds to $\mathrm{We}^{-1} \approx 5$, whereas for $\mathrm{Ri}=1$ the surface tension that maximizes the instability is much smaller: $\mathrm{We}^{-1} \approx 0.2$. However, for both cases, the optimal wave number is $k \approx 0.5$.

In order to determine the optimal parameter configuration for maximum modal growth, we consider contours of maximum growth rate in the $\mathrm{We}^{-1}$-Ri plane in Fig. 5, where both the intensity of the stratification and of the surface tension are varied. We observe the presence of a saddle-shaped region close to $\mathrm{We}^{-1}=5$ and $\mathrm{Ri}=1$ in which the growth rate is significantly enhanced. In particular, the maximum maximorum is located at $\mathrm{We}^{-1}=1.96$ and $\mathrm{Ri}=0.63$ (marked by $\mathbf{X}$ ).

\section{B. Counterpropagating wave dynamics}

Previous studies on interfacial wave interaction showed how different typologies of waves can interact (e.g., Rossby waves [17,19], gravity waves [16], Rossby and capillary waves [8], Alfvén waves [20]). From this perspective, the Kelvin-Helmotz instability can also be interpreted as a wave resonance phenomenon [21,22]. In all these combinations, the interaction leading to instability is mainly between two counterpropagating waves, which are phase locked in a growing configuration. Following Ref. [8], we can "switch off" waves just by eliminating the corresponding row and column in the matrix $\bar{M}$ of Eq. (14). For instance, if the first and fourth rows or columns are eliminated, the 


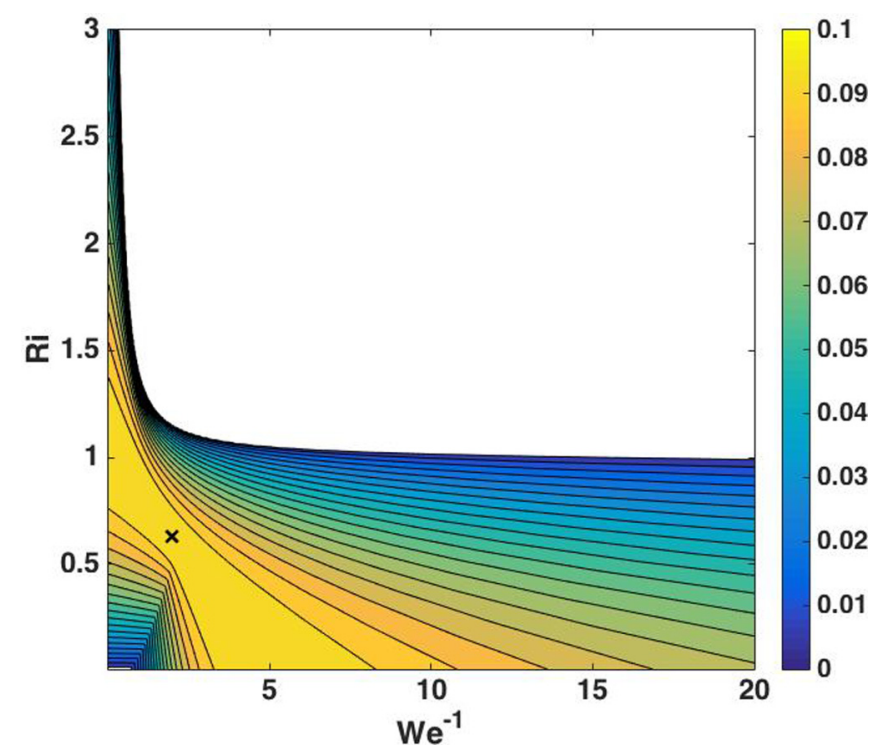

FIG. 5. Maximum growth rate in the $\mathrm{We}^{-1}$-Ri plane. The maximum maximorum is located at $\mathrm{We}^{-1}=1.96$ and $\mathrm{Ri}=0.63$ marked by $\mathbf{X}$.

following dynamical system,

$$
\dot{\mathbf{q}}_{\text {counter }}=\overline{\boldsymbol{M}}_{\text {counter }} \mathbf{q}_{\text {counter }}
$$

where

$$
\overline{\boldsymbol{M}}_{\text {counter }}=i\left[\begin{array}{cc}
-k+\sqrt{\frac{k F}{2}} & R \sqrt{\frac{k F}{2}} \\
-R \sqrt{\frac{k F}{2}} & k-\sqrt{\frac{k F}{2}}
\end{array}\right] .
$$

is obtained. Equation (15) represents a reduced system in which only the two counterpropagating waves are present. The eigenvalues of $\overline{\boldsymbol{M}}_{\text {counter }}$ are the normal modes of this reduced system.

Remembering that $F_{T}=F_{B}$ for $\mathrm{Ri}_{T}=\mathrm{Ri}_{B}$ and $\mathrm{We}_{T}=\mathrm{We}_{B}$, Fig. 6 depicts by a full line the growth rate of the entire system, i.e., the system with all the four waves described by Eq. (14), as compared to the dashed line for the growth rate of the reduced system [Eq. (15)] for the configuration corresponding to the maximum maximorum: $\mathrm{We}^{-1}=1.96$ and $\mathrm{Ri}=0.63$ (see Fig. 5). The agreement is remarkable. Particularly both the maximum growth rate and the cutoff wave number of the entire system are very well approximated by the reduced system. In contrast, if the two counterpropagating waves are switched off, i.e., by eliminating the second and third rows or columns of $\overline{\boldsymbol{M}}$, the resulting system would be composed just of pro-propagating waves whose corresponding normal modes are always neutral.

Figure 6 indicates that the essence of the instability can be obtained when taking into account only the counterpropagating waves, i.e., $\hat{q}_{T}^{-}$and $\hat{q}_{B}^{+}$. This makes sense since their phase speeds without interaction are close to each other, which enables them to resonate. Let us now describe in more detail this resonance. Equation (15) can be written explicitly, using Eqs. (11), as

$$
\begin{aligned}
& \left(\frac{\partial}{\partial t}+i k\right) \hat{q}_{T}^{-}=i \sqrt{\frac{k F}{2}}\left[\hat{q}_{T}^{-}+\hat{q}_{B}^{+} e^{-2 k}\right], \\
& \left(\frac{\partial}{\partial t}-i k\right) \hat{q}_{B}^{+}=-i \sqrt{\frac{k F}{2}}\left[\hat{q}_{T}^{-} e^{-2 k}+\hat{q}_{B}^{+}\right] .
\end{aligned}
$$




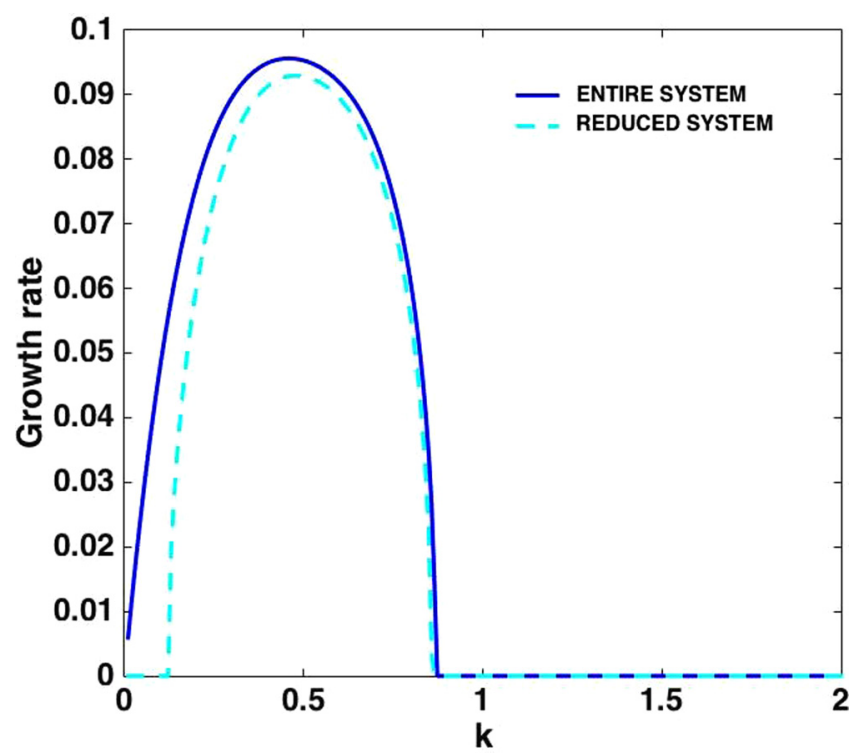

FIG. 6. Comparison between the growth rates of the entire system of the four waves [Eq. (14)], indicated by the continuous line, and the reduced system [Eq. (15)] of the two counterpropagating waves (dashed line) for the configuration corresponding to the maximum maximorum growth at $\left(\mathrm{We}^{-1}=1.96, \mathrm{Ri}=0.63\right)$.

If we introduce the positive waves' amplitudes $Q_{T, B}$ and their phases $\epsilon_{T, B}$, we can write $\hat{q}_{T}^{-}=$ $Q_{T}(t) e^{i \epsilon_{T}(t)}$ and $\hat{q}_{B}^{+}=Q_{B}(t) e^{i \epsilon_{B}(t)}$ and substitute back in Eq. (16) to obtain

$$
\begin{aligned}
\frac{\dot{Q}_{T}}{Q_{T}} & =\chi \frac{Q_{B}}{Q_{T}} \sin \Delta \epsilon, \\
\frac{\dot{Q}_{B}}{Q_{B}} & =\chi \frac{Q_{T}}{Q_{B}} \sin \Delta \epsilon, \\
-\frac{\dot{\epsilon}_{T}}{k} & =\left(1-\sqrt{\frac{F}{2 k}}\right)-\frac{\chi}{k} \frac{Q_{B}}{Q_{T}} \cos \Delta \epsilon, \\
-\frac{\dot{\epsilon}_{B}}{k} & =-\left(1-\sqrt{\frac{F}{2 k}}\right)+\frac{\chi}{k} \frac{Q_{T}}{Q_{B}} \cos \Delta \epsilon,
\end{aligned}
$$

where

$$
\chi=e^{-2 k} \sqrt{\frac{k F}{2}} \quad \text { and } \quad \Delta \epsilon=\epsilon_{\mathrm{T}}-\epsilon_{\mathrm{B}}
$$

are the interaction coefficient and the phase difference, respectively.

Equation set (17) is straightforward to interpret: (17a) and (17b) are the equations for the instantaneous wave growth rates, whereas $(17 \mathrm{c})$ and $(17 \mathrm{~d})$ denote their instantaneous phase speed. Without interaction $(\chi=0)$, the waves are neutral and propagate counter to the interfacial flow according to Eqs. (11a) and (11b). When the interaction is taken into account, Eqs. (17a) and (17b) indicate that when the vorticity phase difference between the waves satisfies $0<\Delta \epsilon<\pi$ the velocity field induced by each wave amplifies the other and makes it grow.

The optimal growth configuration is when $\Delta \epsilon=\frac{\pi}{2}$ [Fig. 7(a)]. Equations (17c) and (17d) indicate further that when $-\frac{\pi}{2}<\Delta \epsilon<\frac{\pi}{2}$ the waves also help each other to counterpropagate while if 

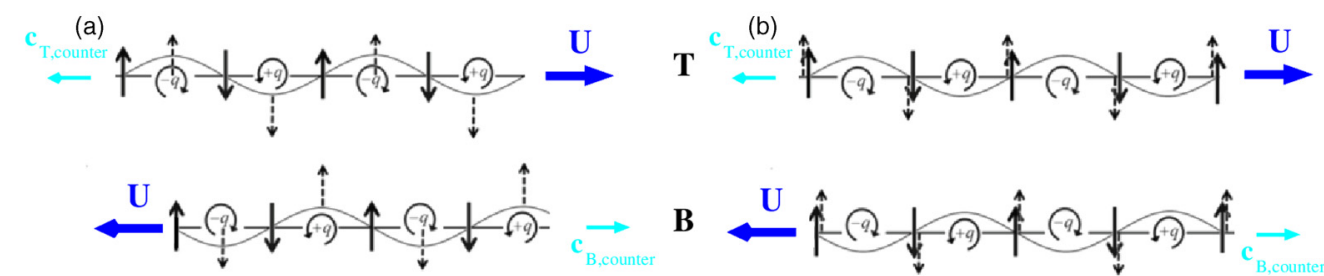

(c)
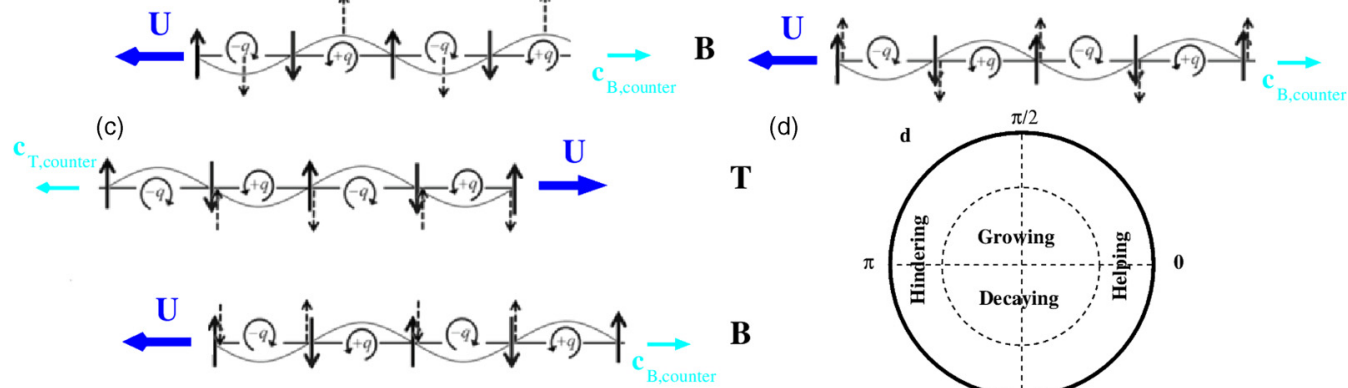

$\mathbf{T}$

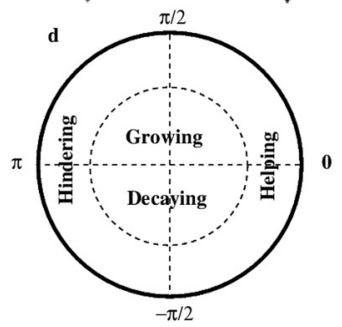

FIG. 7. Schematic description of the interactions between the two interfacial waves as a function of their vorticity phase difference $\Delta \epsilon$. (a) $\Delta \epsilon=\pi / 2$, the cross-stream velocities (dashed arrows) induced by each wave increase the amplitude of the other. (b) $\Delta \epsilon=0$, the cross-stream velocities interfere constructively so that each wave helps the other to propagate faster against its local mean flow. (c) $\Delta \epsilon=\pi$, the cross-stream velocities interfere destructively so that each wave hinders the other counterpropagation rate. (d) Phase diagram of the instantaneous interaction between the waves. When $\pi>\Delta \epsilon>0$, the waves amplify each other, whereas when $-\pi<\Delta \epsilon<0$ they decrease each other's amplitudes. When $-\frac{\pi}{2}<\Delta \epsilon<\frac{\pi}{2}$, the waves help each other to counter-propagate while if $\frac{\pi}{2}<\Delta \epsilon<\frac{3 \pi}{2}$ the waves hinder each other propagation counter their local mean flow.

$\frac{\pi}{2}<\Delta \epsilon<\frac{3 \pi}{2}$ the waves hinder each other's propagation. For instance, when the waves are in phase, $\Delta \epsilon=0$ [Fig. 7(b)], the cross-stream velocity induced by each wave on the opposed one are in superposition. Hence, the interaction does not amplify the waves' amplitudes but rather increases the cross-stream velocity. As indicated from Fig. 2, the wave propagation results from shifting the wave displacement by this cross-stream velocity. Therefore, when the waves reinforce each other's cross-stream velocity, they also increase the propagation velocity of each wave against its local mean flow and hence help each other to resist the shear. In contrast, when the waves are in antiphase [Fig. 7(c)], they hinder each other's propagation rate against the shear. Figure 7(d) summarizes the instantaneous interacting effect between the waves as a function of $\Delta \epsilon$, combining both the amplitude growth or decay as well as helping or hindering the propagation rate. The reader can find more information about the helping and hindering mechanism in Ref. [10].

\section{The normal mode instability as a phase-locking system}

By definition, normal mode instability (of the form of $e^{i k(x-c t)}$ ) is obtained when both waves experience the same growth rate and phase speed. From Eqs. (17a) and (17b), this implies that

$$
k c_{i}=\frac{\dot{Q}_{T}}{Q_{T}}=\frac{\dot{Q}_{B}}{Q_{B}}=\chi \sin (\Delta \epsilon)
$$

and $Q_{T}=Q_{B}$. Furthermore, Eqs. (17c) and (17d) imply that

$$
c_{r}=-\dot{\epsilon}_{T} k=-\dot{\epsilon}_{B} k \text {. }
$$

Thus, in order to have the normal mode instability $\left(Q_{T}=Q_{B}\right.$ and $\left.\sin (\Delta \epsilon)>0\right)$, this gives

$$
\cos (\Delta \epsilon)=\frac{k}{\chi}\left(1-\sqrt{\frac{F}{2 k}}\right) .
$$





FIG. 8. Contours of the phase difference $\frac{\Delta \epsilon}{\pi}$ in the $\left(k-W e^{-1}\right)$ plane for (a) $R i=0.5$, (b) $R i=1$ and (c) $R i=2$, and (d) in the ( $k-\mathrm{Ri})$ plane for $\mathrm{We}^{-1}=0$.

From the last equation, we can find that

$$
\text { (hindering) } \quad-1<\frac{k}{\chi}\left(1-\sqrt{\frac{F}{2 k}}\right)<1 \quad \text { (helping). }
$$

If we refer to Eq. (22) for the phase and Eq. (18) for $\chi$, and take only the positive angle (relevant to growth), the condition for instability becomes

$$
\text { (hindering) } 0<\arccos \left[e^{2 k}\left(1-\sqrt{\frac{F}{2 k}}\right)\right]<\pi \quad \text { (helping). }
$$

In Figs. 8(a)-8(c), the phase difference, rescaled by $\pi,\left(\frac{\Delta \epsilon}{\pi}\right)$ is illustrated in the $\left(k-\mathrm{We}^{-1}\right)$ plane for the same configurations depicted in Figs. 4(a)-4(c): $\mathrm{Ri}=0.5, \mathrm{Ri}=1$, and $\mathrm{Ri}=2$, respectively. It is clear that when the instability is active, the phase difference is in the region $0<\Delta \epsilon<\pi$, where the growth is favored. The hindering and helping limit, indicated by arrows, corresponds to the limit of the unstable region. In particular, the largest values of the growth rate correspond to values at the vicinity of $\Delta \epsilon=\frac{\pi}{2}$, which is the instantaneous optimal configuration for growth [16]. We note that 
for pure gravity waves $(F=\mathrm{Ri})$, we get

$$
0<\arccos \left[e^{2 k}\left(1-\sqrt{\frac{\mathrm{Ri}}{2 k}}\right)\right]<\pi
$$

As indicated by Eq. (12), as the wave number increases, the propagation rate decreases. Hence the hindering (helping) limit is obtained for small (large) wave numbers. Both the hindering and helping limits are shown in Fig. 8(d), where the contours of the phase difference $\frac{\Delta \epsilon}{\pi}$ are depicted in the $(k-\mathrm{Ri})$ plane in the absence of surface tension.

Finally, by combining Eqs. (19) and (23) together we retrieve the expression for the modal growth rate,

$$
k c_{i}= \pm \chi\left[1-\cos ^{2}(\Delta \epsilon)\right]^{\frac{1}{2}}= \pm\left[\frac{k F}{2}\left(\frac{e^{-4 k}}{4}-1\right)-k^{2}+k \sqrt{2 k F}\right]^{\frac{1}{2}}
$$

which is nothing more than the real part of the eigenvalues of $\overline{\boldsymbol{M}}_{\text {counter }}$ defined in Eq. (15), when non-null.

It is possible to calculate also the maximum growth rate of the reduced system with respect to the wave number. Remembering that $F=\mathrm{Ri}+k \mathrm{We}^{-1}$, we obtain

$$
\frac{\partial\left(k c_{i}\right)}{\partial k}=\frac{\frac{\mathrm{Ri}+3 k^{2} \mathrm{We}^{-1}}{2}\left(\frac{e^{-4 k}}{4}-1\right)-k F \frac{e^{-4 k}}{2}-2 k+\frac{k\left(3 \mathrm{Ri}+5 k^{2} \mathrm{We}^{-1}\right)}{\sqrt{2 k F}}}{2 \sqrt{\frac{k F}{2}\left(\frac{e^{-4 k}}{4}-1\right)-k^{2}+k \sqrt{2 k F}}} .
$$

In Fig. 9(a), the contours of this estimated maximum are depicted in the $\left(\mathrm{Ri}^{-\mathrm{We}^{-1}}\right)$ plane. We notice that there is a central region where a maximum maximorum is present. This is in agreement with the position of the maximum of the growth rate for the entire system; see Fig. 5. A more qualitative comparison is achieved in Fig. 9(b). In this figure, the contours of the maximum growth rate for the entire system are depicted in the $\left(\mathrm{Ri}-\mathrm{We}^{-1}\right)$ plane by the color label, while the contours of the maximum for the reduced system by black dashed lines. The agreement is both qualitatively and quantitatively satisfying in the region of maximum instability.

However, there is an increasing discrepancy between the two systems as the capillary force strengthens. In particular, the reduced system underestimates the instability growth, indicating that
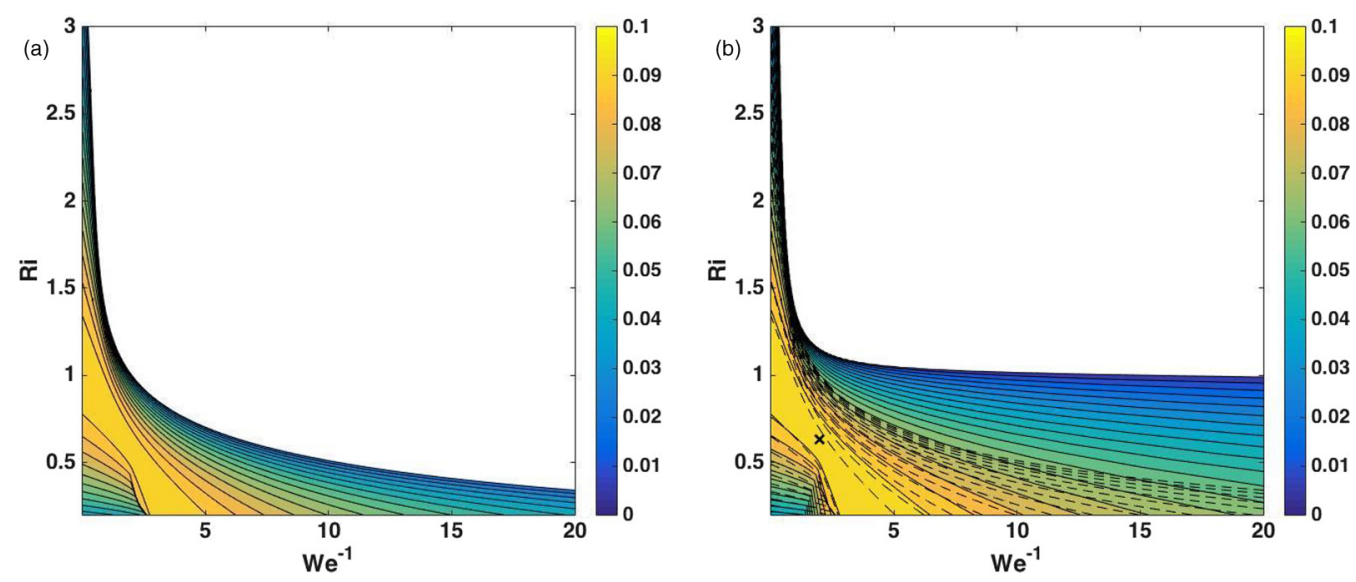

FIG. 9. (a) Contours of the maximum growth rate for the reduced system, i.e., with considering just the two counterpropagating waves in the plane $\mathrm{Ri}-\mathrm{We}^{-1}$. (b) Comparison between the maximum growth rate for the entire system, i.e., considering all the four waves, and the reduced system. The entire system is depicted by the color label, while the reduced system is shown by dashed lines. 
the pro-propagating capillary waves play a non-negligible role in the destabilization. This stands in contrast to the results found for interfacial gravity waves [16]. The fundamental difference between interfacial gravity and capillary waves, in terms of wave interaction, lies in the dependence of their intrinsic phase speeds on the wave number $k$. Surface tension is more efficient at small scales whereas buoyancy is more efficient at larger scales. As indicated from Eq. (12), the interfacial intrinsic wave speed grows indeed with the wave number due to the surface tension restoring force but decreases with the wave number due to the buoyancy. On the other hand, the wave interaction coefficient $\chi$ in Eq. (18) always decreases with the wave number. Hence, for small wave numbers, where the wave interaction is strong the contribution of the surface tension to the propagation relative to the mean flow (both counter and pro) is relatively small. Therefore, both the counter- and the pro-propagating capillary waves are expected to contribute to the instability. In contrast, at small wave numbers, the pure gravity phase speed relative to the mean flow is large, and thus we expect that only the counterpropagating waves will contribute to the instability mechanism.

\section{A PURE CAPILLARY INSTABILITY}

In this section, we suppress the stratification, i.e., $\mathrm{Ri}=0$, and focus on the pure capillary wave instability. In Sec. V A, we analyze the linear stability, while in Sec. V B, we perform fully nonlinear DNS illustrating the nonlinear evolution of this instability.

\section{A. Linear stability analysis}

Figure 10 is the same as Fig. 3 but for Ri $=0$. It is clear that the instability can be maintained solely by counterpropagating capillary waves. As in Sec. IV, the resonance instability appears when the two $c_{\text {counter }}$ have equal velocities, here $c_{T}^{-}=c_{B}^{+}=0$. This is attained in the vicinity of $k=\frac{2}{W e^{-1}}$. In particular, the maximum growth rate is initially increasing as surface tension grows and then decreases. In contrast, the unstable range of wave numbers is shifting monotonously toward high wavelengths as the surface tension grows. The destabilization due to the surface tension is manifested in the $\left(k-\mathrm{We}^{-1}\right)$ plane in Fig. 11(a). The maximum growth rate $k c_{i}=0.095$ is around $k=0.5$ and $\mathrm{We}^{-1}=5$. The unstable wave-number region is centered around the $\mathrm{We}^{-1}=\frac{2}{k}$ curve since this is the wave number for which the two $c_{\text {counter }}$ are equal [see the dots in Fig. 10(d)].

As for the mixed gravity-capillary waves, the maximum values of the growth rate correspond to values near the phase difference $\Delta \epsilon=\frac{\pi}{2}$ [Fig. 11(b)]. The unstable region is bounded as well by the hindering and the helping limit; however, for pure capillary waves $\left(F=\mathrm{We}^{-1} k^{2}\right)$, as the wave number increases so does the propagation rate. Thus, the hindering (helping) limit is obtained for large (small) wave numbers. This is opposite to pure gravity waves instability [Fig. 8(d)]. The interaction coefficient $\chi$ [Eq. (18)] has also different dependencies on the wave number: $\chi_{g r}=e^{-2 k} \sqrt{\frac{k \mathrm{Ri}}{2}}$, $\chi_{\text {cap }}=e^{-2 k} \sqrt{\frac{k^{3} \mathrm{We}^{-1}}{2}}$, for pure gravity and capillary waves, respectively.

\section{B. Nonlinear simulations}

In this section, we conduct DNS to quantify the effects of nonlinearity and viscosity on the pure capillary instability observed in Sec. V A. In particular, in Sec. V B 1 we describe (i) the governing equations and (ii) the numerical setup of the simulations, whereas in Sec. V B 2 we show the results of the DNS.

\section{DNS: Governing equations, numerical method, and setup}

We have conducted DNS of the setup shown in Fig. 1 to unveil the nonlinear evolution of the pure capillary instability. Note that the stratification has been neglected, i.e., $\rho_{1}=\rho_{2}=\rho_{3}$. This means that the fluid at the top (fluid 1) is equal to the fluid at the bottom (fluid 3 ), since we consider also the same strength of the surface tension at the two interfaces. For this reason, we will refer simply to fluid 1 (fluid 2) for the outer fluids (inner fluid) from now on. 

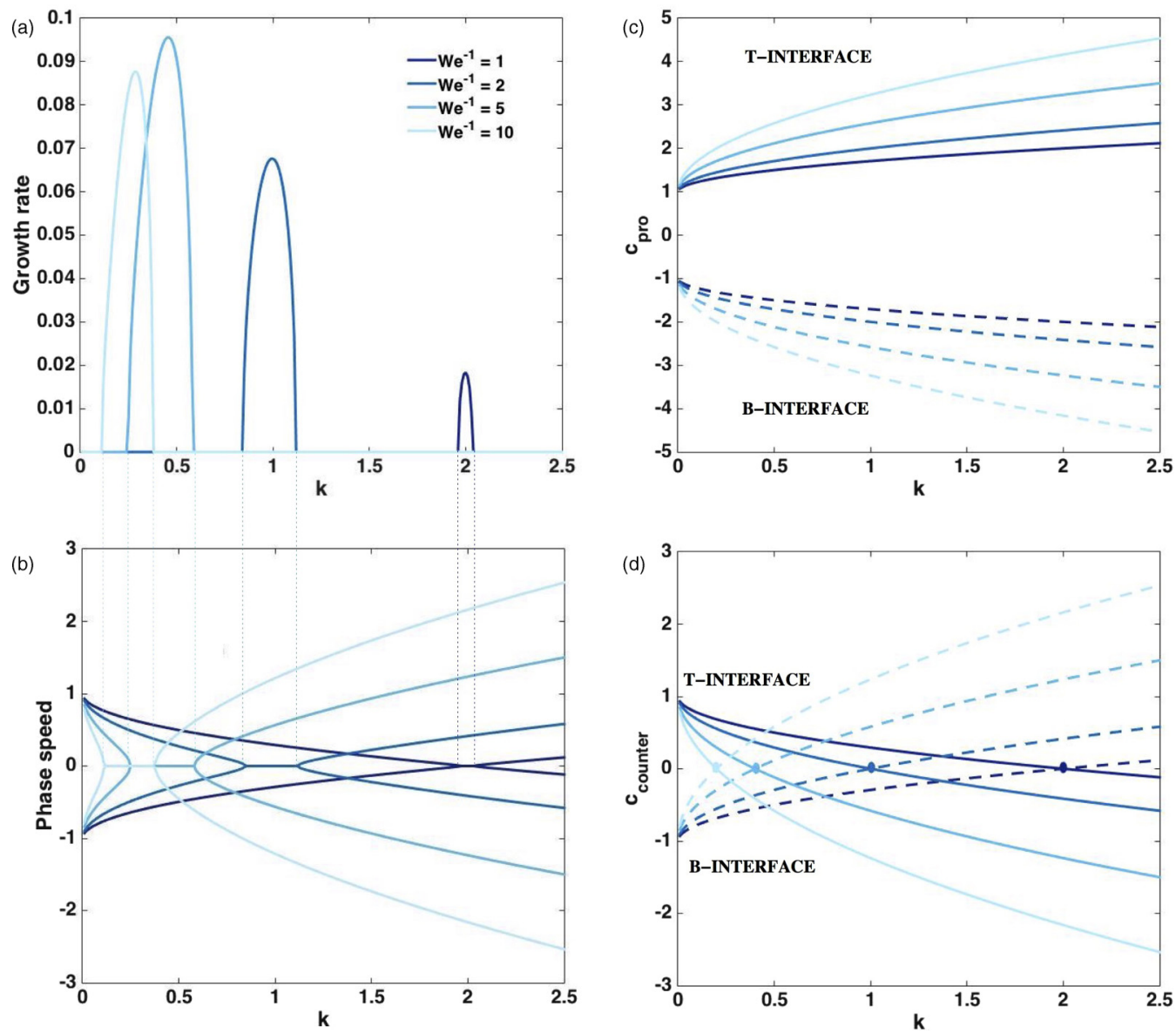

FIG. 10. Same as in Fig. 3 but for $\mathrm{Ri}=0$.

The governing equations are the incompressible dimensionless Navier-Stokes equations

$$
\begin{aligned}
\frac{\partial \mathbf{u}}{\partial t}+(\mathbf{u} \cdot \nabla) \mathbf{u} & =\nabla p+\frac{1}{\operatorname{Re}} \Delta \mathbf{u}, \\
\nabla \cdot \mathbf{u} & =0,
\end{aligned}
$$

where $\mathbf{u}=(u, v)$ is the velocity field, $t$ is the time, $p$ is the pressure, and $\operatorname{Re}=\frac{U_{y} h^{2}}{v}$ is the Reynolds number based on half the distance between the interfaces and the upper interface velocity $U_{I}=U_{y} h$. The boundary conditions at the interfaces $T$ and $B$ between the fluids read (i) continuity of velocity at the interfaces $[[\mathbf{u}]]_{T, B}=0$, (ii) kinematic conditions $\frac{\partial \mathcal{D}_{T, B}}{\partial t}+\frac{\partial u}{\partial x} \frac{\partial \mathcal{D}_{T, B}}{\partial x}=\frac{\partial u}{\partial y}$, where $y=\mathcal{D}_{T, B}(x, t)$ is the respective location of the top $T$ and bottom $B$ interfaces, (iii) jump of the stress due to the surface tension $\left[\left[\sigma \cdot \mathbf{n}_{T, B}\right]\right]_{T, B}=\frac{1}{\mathrm{We}} \kappa_{T, B} \mathbf{n}_{T, B}$, where $\mathbf{n}_{T, B}$ is the normal to the interfaces $\mathbf{n}_{T, B}=$ $\frac{1}{\sqrt{\left(\frac{\partial \mathcal{D}_{T, B}}{\partial x}\right)^{2}+1}}\left(-\frac{\partial \mathcal{D}_{T, B}}{\partial x}, 1\right)$ and $\kappa_{T, B}=\nabla \cdot \mathbf{n}_{T, B}$ is the curvature.

The numerical tool used to conduct the numerical simulations is OPENFOAM [23]. This tool is a flexible open-source code which accurately solves the Navier-Stokes equations by a finite volume method [24]. Particularly we used the multiphase solver MULTIPHASEINTERFOAM which is part of the INTERFOAM solver family. These solvers have given accurate results in surface-tension-dominated 

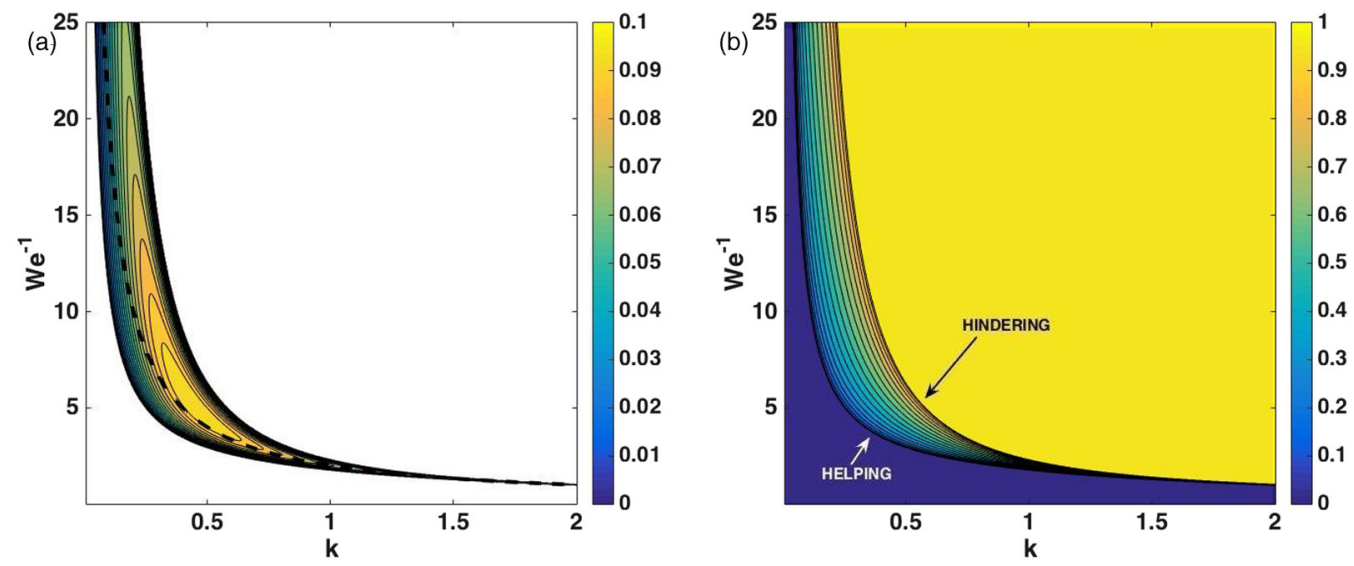

FIG. 11. Pure capillary setup: contours of (a) the growth rate and (b) the phase difference $\frac{\Delta \epsilon}{\pi}$ in the plane $k-\mathrm{We}^{-1}$.

flows [25]. The temporal derivatives are discretized by using the implicit Euler scheme, while spatial derivatives are discretized by using mainly second-order-accurate schemes (central differencing and Van Leer scheme [26] for the concentration advective terms). The only exceptions are the advective terms of the velocity for which a straightforward upwind scheme is implemented. The INTERFOAM family uses the Multidimensional Universal Limiter with Explicit Solution (MULES) [25] solver to treat the interfaces (where $\alpha_{1,2}$ denotes the concentration of each fluid). MULES is part of the volume-of-fluid methods family [27]. Finally, the surface tension is modeled by the continuum surface force method [28].

The sizes of the two-dimensional computational domain are $L_{x}=\frac{4 \pi h}{k_{\max }}$ and $L_{y}=2.5$ in the streamwise $(x)$ and wall-normal ( $y$ ) directions, respectively. Recall that $k_{\max }$ is the capillary instability maximal wave number corresponding to $\mathrm{We}^{-1}=5$ [see Fig. 11(a)]. The value of the horizontal length $L_{x}$ was chosen in order to contain two wavelengths of the most unstable pure capillary instability mode in the streamwise direction, whereas the height was chosen large enough to avoid any confinement effect on the flow. Because of the choice of the horizontal length, we can capture the instabilities with a wave number $k \geqslant \frac{k_{\max }}{2}$. The distance of the two interfaces was set to $2 h=0.2$. The initial velocity profile is $U=\left[U_{y} y+u_{d}, v_{d}\right]$ where a sinuous disturbance

$$
\begin{aligned}
& u_{d}=C \frac{100 y}{L_{y}^{2}} e^{-\left(\frac{10 y}{L_{y}}\right)^{2}} \sin \left(\frac{k_{\max } x}{h}\right), \\
& v_{d}=C e^{-\left(\frac{10 y}{L_{y}}\right)^{2}} \cos \left(\frac{k_{\max } x}{h}\right),
\end{aligned}
$$

is introduced with $C=0.001$. The disturbance wavelength is again equal to $\lambda_{d}=\frac{2 \pi}{k_{\max }}$ in order to easily trigger the pure capillary instability at $\mathrm{We}^{-1}=5$. The initial concentrations of the two fluids 1 and 2 were $\alpha_{1}=1$ and $\alpha_{2}=0$ for $|y|>h$, while for $|y|<h \alpha_{1}=0$ and $\alpha_{2}=1$.

Periodic boundary conditions have been implemented along the $x$ direction, whereas no-slip boundary conditions with settling the with wall velocity $u\left( \pm \frac{L_{y}}{2}\right)= \pm \frac{L_{y}}{2}$ are chosen along the $y$ direction. The number of grid points are settled to $N_{x}=150$ and $N_{y}=150$ along the streamwise and wall-normal directions, respectively. However, we have conducted tests with a finer grid, i.e., with the double of grid points along both directions. No significant discrepancies in the results were observed. 

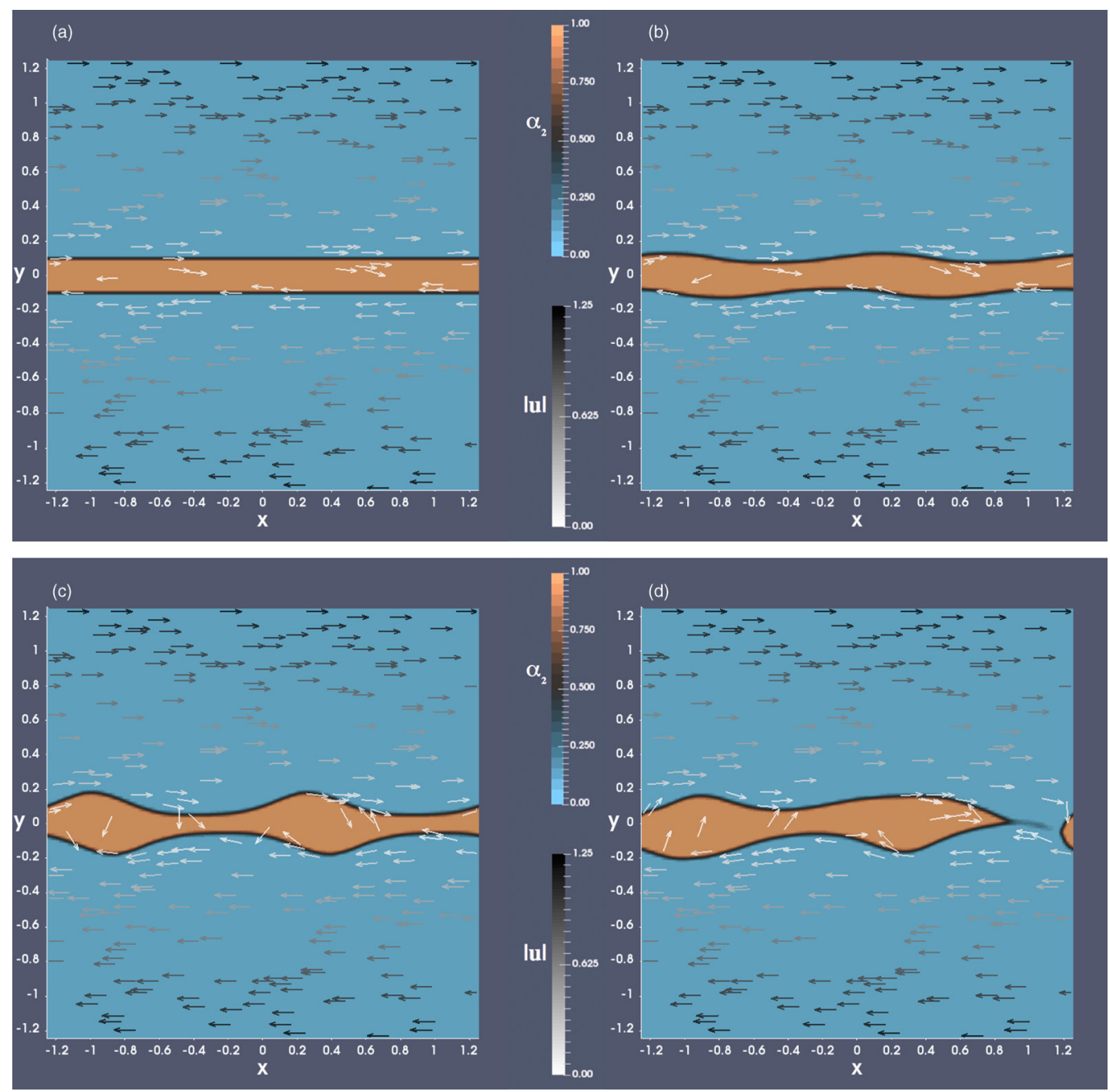

FIG. 12. Fields of the concentration of the fluid $2\left(\alpha_{2}\right)$ in the plane $x-y$ for $\operatorname{Re}=10^{7}$ and $\mathrm{We}^{-1}=5$ at different times (a) $t=0$, (b) $t=18.75$, (c) $t=75$, and (d) $t=133.25$. The color (not the length) of the arrows in grayscale depicts the velocity magnitude.

\section{Stability analysis}

In this section, we examine the DNS results. In Fig. 12, we report in color labels the fields of the concentration of the fluid $2 \alpha_{2}$ in the plane $x-y$ taken at different times: (a) $t=0$, (b) $t=18.75$, (c) $t=75$, and (d) $t=133.25$, for $\mathrm{Re}=10^{7}$ and $\mathrm{We}^{-1}=5$. The arrows in grayscale depict the velocity magnitude, clearly showing the presence of the shear and the direction of the velocity. It should be noticed that for $\mathrm{Re}=10^{7}$ the viscous effects can be neglected, allowing us to directly compare the results with the inviscid linear theory. The instability triggered by the initial disturbance grows [Figs. 12(b) and 12(c)] until breaking up the interfaces and forming a drop [Fig. 12(d)]. Moreover, the wavelength corresponds to $k_{\max }$ since we have two periods of the mode along the streamwise direction. This shows that flow is destabilized by surface tension and confirms the existence of the pure capillary mode when restoring nonlinearity.

In Fig. 13(a), the evolution of the rms-streamwise velocity disturbance $u_{r m s}$ is shown for Re $=10^{7}$ and different values of $\mathrm{We}^{-1}$. The root mean square (rms) of a streamwise velocity is defined by 

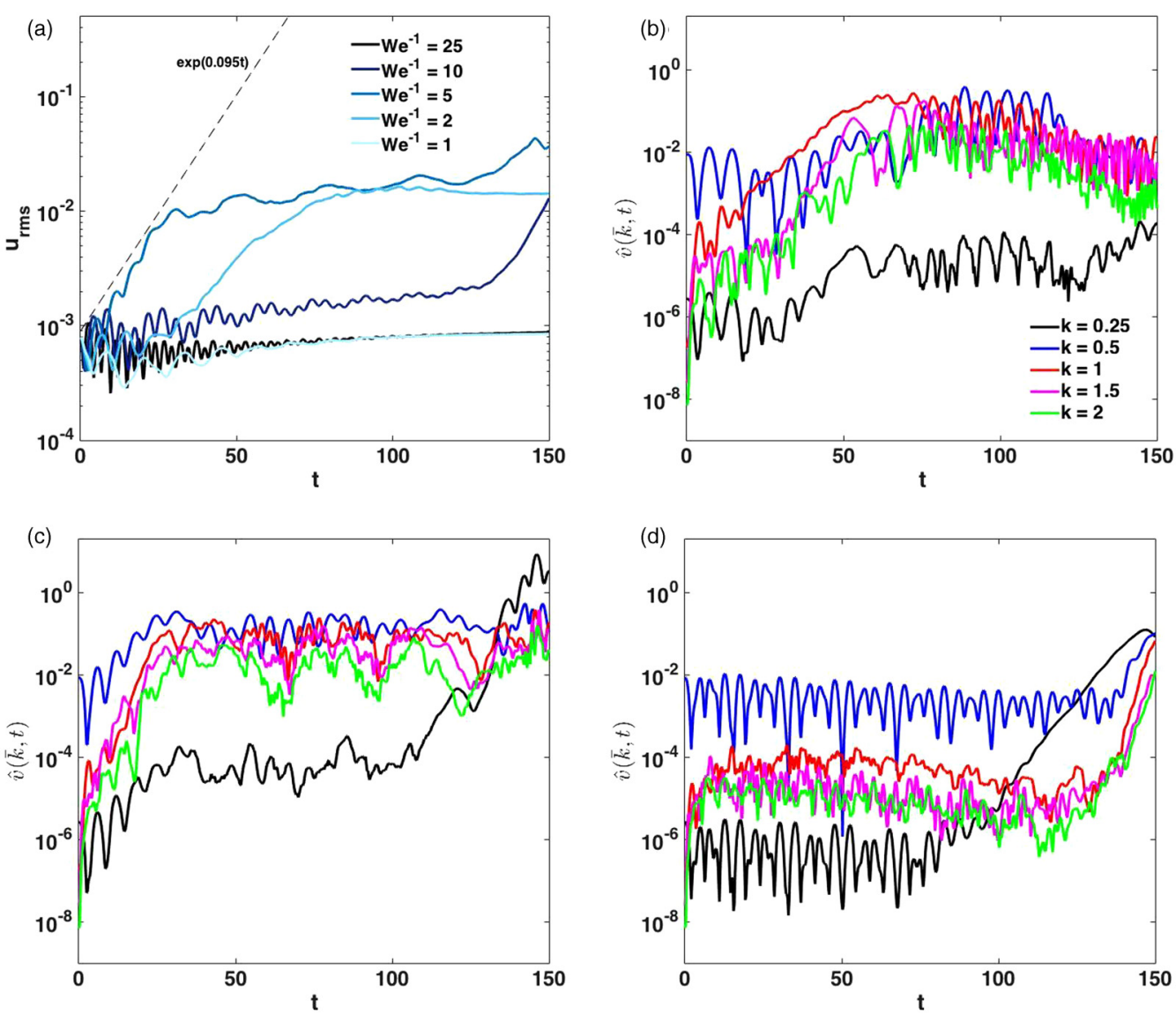

FIG. 13. Temporal evolution of (a) the rms-streamwise velocity disturbance $u_{r m s}$ for different values of the surface tension $\mathrm{We}^{-1}$ at $\operatorname{Re}=10^{7}$ and $[(\mathrm{b})-(\mathrm{d})] \hat{v}(\bar{k})$ with $k$ equal to $k=0.25$ (black line), $k=0.5$ (blue), $k=1$ (red), $k=1.5$ (magenta), and $k=2$ (green) for (b) $\mathrm{We}^{-1}=2$, (c) $\mathrm{We}^{-1}=5$, and (d) $\mathrm{We}^{-1}=10$.

its integral in space: $u_{r m s}=\sqrt{\overline{u^{2}}}$ where $\bar{u}=\int_{-\frac{L_{y}}{2}}^{\frac{L_{y}}{2}} \int_{-\frac{L_{x}}{2}}^{\frac{L_{x}}{2}}\left[\mathbf{u}(x, y)-U_{y} y\right] d x d y$. If the configuration is unstable, the mode energy is growing until reaching a nonlinear state corresponding to the interface breakup.

One can observe how the flow is initially destabilized when increasing the surface tension, but the instability is gradually damped and finally suppressed when the influence of the surface tension is too strong. This is the same behavior observed in Sec. V A. In particular, the range of the Weber numbers for which the flow is unstable is between $2 \leqslant \mathrm{We}^{-1} \leqslant 10$. While for $\mathrm{We}^{-1}=25$, the absence of instability is consistent with the linear analysis [see Fig. 11(a)] since this case is stable for $k \geqslant \frac{k_{\max }}{2}$, for $\mathrm{We}^{-1}=1$, DNS does not seem fully in agreement with the linear stability analysis. For this case, there is a very small range of unstable wave numbers around $k=2$ [see Fig. 11(a)]. However, the instability is probably too weak to be properly appreciable in the DNS. However, the strongest perturbation growth is obtained for $\mathrm{We}^{-1}=5$ as predicted by the linear theory for $k>\frac{k_{\max }}{2}$. Furthermore, the initial linear growth for $\mathrm{We}^{-1}=5$ is relatively close to the value predicted by linear theory $k c_{i}=0.095$. For $\mathrm{We}^{-1}=2$ and $\mathrm{We}^{-1}=10$, the exponential growth is not immediate but starts after a certain amount of time. This occurs since the initial disturbance is not unstable for these cases; i.e., we do not immediately perturb the wave number corresponding to the maximum growth rate for these configurations but $k=0.5$ which is stable [see Fig. 11(a)]. However, the initial 




FIG. 14. Temporal evolution of the rms-streamwise velocity disturbance $u_{r m s}$ for different values of the Reynolds number $\mathrm{Re}$ at $\mathrm{We}^{-1}=5$.

disturbance eventually perturbs other wavelengths triggering an instability with a wave number $k \neq k_{\max }$.

We calculate the $y$-integrated Fourier transform along $x$ of the vertical velocity $\hat{v}(k, t)$ to observe in detail which wavelengths are triggered. In Figs. 13(b)-13(d), we illustrate the temporal evolution of $\hat{v}(k, t)$ for several wave numbers $k$ for (b) $\mathrm{We}^{-1}=2$, (c) $\mathrm{We}^{-1}=5$, and (d) $\mathrm{We}^{-1}=10$. One can notice that the dominant growing mode changes for the three different values of the surface tension. In particular, for $\mathrm{We}^{-1}=5$ the instability is triggered by $k=k_{\max }=0.5$ (blue line), as expected. For $\mathrm{We}^{-1}=2$, the wave number of the dominant mode is $k=1$ (red line), which is consistent with the linear stability analysis since the most unstable mode is around $k=1$ [Fig. 11(a)]. Absent in the initial condition, it is soon triggered by nonlinear harmonic generation. For $\mathrm{We}^{-1}=10$, the dominant wave number is $k=0.25$ (black line), again in agreement with the linear stability. Also absent in the initial condition, it is subharmonically generated only after a much longer transient.

Finally, we introduce viscous effects into the numerical simulations by reducing the Reynolds number. The evolution of the rms-streamwise velocity disturbance $u_{r m s}$ for these simulations is reported in Fig. 14 with settling $\mathrm{We}^{-1}=5$. Viscous effects start to play a role for $\operatorname{Re}=10^{5}$, while they remain negligible above this value. We observe that the viscosity progressively damps the pure capillary instability, eventually quenching it for $\operatorname{Re}=10^{3}$. It should be noticed that for $\mathrm{Re}=10^{4}$ the interface is not breaking up as occurred for inviscid flows once we reach saturation (not shown).

\section{CONCLUSIONS}

In this work, we have analyzed by means of the KW perspective the normal mode inviscid instability of a setup à la Taylor-Caulfield including capillary effects. With this perspective, we have seen how two capillary-gravity waves form at the interfaces. These waves can interact between each other, generating a Taylor-Caulfield instability modified by surface tension.

The inclusion of capillary effects is the main contribution of this work. In particular, we have found that capillarity destabilizes the Taylor-Caulfield instability for intermediate values of the surface tension. The capillary-gravity waves interact similarly to the pure gravity waves [16]: 
(i) the counterpropagating waves can phase lock to generate the instability and (ii) their mutual growth occurs for a phase difference of $0<\Delta \epsilon<\pi$. These characteristics explain the position of the maximum maximorum of the growth rate at $\mathrm{We}^{-1}=1.96$ and $\mathrm{Ri}=0.63$.

Interestingly, the instability persists if the stratification is suppressed. Therefore, a pure capillary instability, i.e., an instability sustained just by the interaction of the counterpropagating capillary waves, is generated. As far as we know, there is no mention of a similar instability in the existing literature. We have afterward compared the linear stability results to ad hoc inviscid direct numerical simulations conducted with OPENFOAM. The maximum of the disturbance growth in the simulations is found for $\mathrm{We}^{-1}=5$, in agreement with the linear stability analysis. Furthermore, the value of the growth rate is very similar in the two analyses. This confirms the quality of the predictions of the linear stability analysis. Finally, viscous effects have been introduced in the simulations, keeping constant the value of the surface tension at $\mathrm{We}^{-1}=5$. A decrease in the Reynolds number damps gradually the instability until quenching it for $\mathrm{Re}=10^{3}$.

The results contained in this paper give further insights into the counterintuitive destabilizing influence of surface tension in planar shear flows [5-8]. Aside from the inherent theoretical aspects, this could be helpful in understanding the effect of capillarity in systems where two gravity (or centrifugal) waves are interacting. For instance, the experiments in swirling rotating tanks show evidence on asymmetric capillary waves which are super-imposed on top of the surface centrifugal waves [29]. Furthermore, it would be interesting to study if there is a connection between the pure capillarity instability described in the present paper with the capillaries instabilities found in core-annular flows [30,31], keeping in mind that the role of viscosity stratification cannot be neglected in this kind of flow.

The DNS setup was build to analyze instabilities with wave number $k \geqslant 0.25$. Moreover, we have introduced a disturbance with wave number $k=0.5$ that corresponds to the optimal value to maximize the pure capillary instability when the surface tension is $\mathrm{We}^{-1}=5$. Viscosity could, however, modify the value of this optimal wave number as well as the optimal value of the surface tension. Our findings then are valid just for this specific configuration. Future works will be devoted to understanding if the pure capillary instability can be found also for lower values of the Reynolds number, where often capillary effects are more important. This points out the need to introduce in a simple way the viscosity in the KW perspective. However, to this goal the dispersion relation can be obtained as well by matching the boundary conditions at the interfaces, as usual in normal mode analysis [2,32].

Furthermore, the interaction of the continuous spectrum of the Couette flow with the interfacial waves considered here should be considered to determine if, similar to Miles's mechanism for gravity wave amplification under the action of wind at the ocean surface [33,34], it can give rise to other instability modes. Additionally, it may lead to transient growth, which may be larger than the fastest growth rate found here. The optimal evolution of the complete set of the system (discrete plus continuous modes) via singular value decomposition is a subject of future work.

In order to observe this pure capillary instability experimentally, in view of the experimental difficulties to realizing a pure Couette flow, the simplest setup appears probably to be a Taylor-Couette apparatus operating in the centrifugal stable regime (rotating inner wall). If the gap is small enough, curvature effects might be expected to not significantly affect the dynamics. Still, the experimental realization hinges on several difficulties. First of all, for this instability not to be masked by viscous interfacial waves [35], three isoviscous fluids are required, thereby ensuring uniform shear across the three layers. Second, to ensure the radial stratification of the fluids, they should be isodense. Third, our simulations show that Reynolds numbers as large as a few thousand are required for the pure capillary instability to overcome viscous effects. At such Reynolds numbers, transition to turbulence may likely not be avoidable, resulting from several transient growth mechanisms and the existence of unstable nonlinear orbits and localized solutions. Finally, the presence of the boundaries in the experimental setup could modify the pure capillary instability characteristics. However, confinement can be easily implemented in the models presented in this paper. It would be enough to change the Green's function [Eq. (5)] as explained by Heifetz and Methven [36]. 
[1] R. A. Gerwin, Stability of the interface between two fluids in relative motion, Rev. Mod. Phys. 40, 652 (1968).

[2] L. Biancofiore and F. Gallaire, Influence of confinement on temporal stability of plane jets and wakes, Phys. Fluids 22, 014106 (2010).

[3] L. Rayleigh, On the instability of jets, Proc. London Math. Soc. 10, 4 (1878).

[4] J. Eggers and E. Villermaux, Physics of liquid jets, Rep. Prog. Phys. 71, 036601 (2008).

[5] O. Tammisola, F. Lundell, and L. D. Soderberg, Effect of surface tension on global modes of confined wake flows, Phys. Fluids 23, 014108 (2011).

[6] O. Tammisola, F. Lundell, and L. D. Soderberg, Surface tension-induced global instability of planar jets and wakes, J. Fluid Mech. 713, 632 (2012).

[7] L. Biancofiore, F. Gallaire, P. Laure, and E. Hachem, Direct numerical simulations of two-phase immiscible wakes, Fluid Dyn. Res. 46, 041409 (2014).

[8] L. Biancofiore, F. Gallaire, and E. Heifetz, Interaction between counterpropagating rossby waves and capillary waves in planar shear flows, Phys. Fluids 27, 044104 (2015).

[9] F. P. Bretherton, Baroclinic instability and the short wavelength cut-off in terms of potential vorticity, Quart. J. Royal Meteorol. Soc. 92, 335 (1966).

[10] B. J. Hoskins, M. E. McIntyre, and A. W. Robertson, On the use and significance of isentropic potential vorticity maps, Quart. J. Royal Meteorol. Soc. 111, 877 (1985).

[11] J. Holmboe, On the behaviour of symmetric waves in stratified shear layers, Geophys. Publ. 24, 67 (1962).

[12] O. M. Umurhan and E. Heifetz, Holmboe modes revisited, Phys. Fluids 19, 064102 (2007).

[13] G. I. Taylor, Effect of variation in density on the stability of superposed streams of fluid, Proc. R. Soc. London, Ser. A 132, 499 (1931).

[14] C.-C. P. Caulfield, Multiple linear instability of layered stratified shear flow, J. Fluid Mech. 258, 255 (1994).

[15] N. Harnik, E. Heifetz, O. M. Umurhan, and F. Lott, A buoyancy-vorticity wave interaction approach to stratified shear flow, J. Atmos. Sci. 65, 2615 (2008).

[16] A. Rabinovich, O. M. Umurhan, N. Harnik, F. Lott, and E. Heifetz, Vorticity inversion and action-at-adistance instability in stably stratified shear flow, J. Fluid Mech. 670, 301 (2011).

[17] E. Heifetz, C. H. Bishop, and P. Alpert, Counter-propagating Rossby waves in the barotropic rayleigh model of shear instability, Quart. J. Royal Meteorol. Soc. 125, 2835 (1999).

[18] D. J. Acheson, Elementary Fluid Dynamics (Oxford University Press, Oxford, UK, 1990).

[19] L. Biancofiore and F. Gallaire, Counterpropagating Rossby waves in confined plane wakes, Phys. Fluids 24, 074102 (2012).

[20] E. Heifetz, J. Mak, J. Nycander, and O. M. Umurhan, Interacting vorticity waves as an instability mechanism for magnetohydrodynamic shear instabilities, J. Fluid Mech. 767, 199 (2015).

[21] A. D. D. Craik, Wave Interactions and Fluid Flows (Cambridge University Press, Cambridge, UK, 1988).

[22] E. Heifetz, Y. Reuveni, A. Gelfgat, E. Kit, and J. Methven, The counterpropagating Rossby wave perspective on Kelvin Helmholtz instability as a limiting case of a Rayleigh shear layer with zero width, Phys. Fluids 18, 018101 (2006).

[23] H. G. Weller, G. Tabor, H. Jasak, and C. Fureby, A tensorial approach to computational continuum mechanics using object-oriented techniques, Comput. Phys. 12, 620 (1998).

[24] F. Moukalled, L. Mangani, and M. Darwish, The Finite Volume Method in Computational Fluid Dynamics: An Advanced Introduction with OpenFOAM ${ }^{\circledR}$ and Matlab, Fluid Mechanics and its Applications, Vol. 113 (Springer, Berlin, 2015).

[25] S. S. Deshpande, L. Anumolu, and M. F. Trujillo, Evaluating the performance of the two-phase flow solver interfoam, Comput. Sci. Disc. 5, 014016 (2012).

[26] B. Van Leer, Towards the ultimate conservative difference scheme, V: A second-order sequel to Godunov's method, J. Comput. Phys. 32, 101 (1979).

[27] R. Scardovelli and S. Zaleski, Direct numerical simulation of free-surface and interfacial flow, Annu. Rev. Fluid Mech. 31, 567 (1999).

[28] J. U. Brackbill, D. B. Kothe, and C. Zemach, A continuum method for modeling surface tension, J. Comput. Phys. 100, 335 (1992). 
[29] L. Tophøj, J. Mougel, T. Bohr, and D. Fabre, Rotating Polygon Instability of a Swirling Free Surface Flow, Phys. Rev. Lett. 110, 194502 (2013).

[30] D. D. Joseph and Y. Renardy, Fundamentals of Two-Fluid Dynamics, Part I: Mathematical Theory and Applications, Interdisciplinary Applied Mathematics, Vol. 3 (Springer Science \& Business Media, Berlin, 1993).

[31] D. D. Joseph, R. Bai, K. P. Chen, and Y. Renardy, Core-annular flows, Annu. Rev. Fluid Mech. 29, 65 (1997).

[32] P. G. Drazin, Introduction to Hydrodynamic Instability (Cambridge University Press, Cambridge, 2002).

[33] J. W. Miles, On the generation of surface waves by shear flows, J. Fluid Mech. 3, 185 (1957).

[34] J. R. Carpenter, A. Guha, and E. Heifetz, A physical interpretation of the wind-wave instability as interacting waves, J. Phys. Oceanogr. 47, 1441 (2017).

[35] F. Charru, Hydrodynamic Instabilities, Cambridge Texts in Applied Mathematics, Vol. 37 (Cambridge University Press, Cambridge, UK, 2011).

[36] E. Heifetz and J. Methven, Relating optimal growth to counterpropagating Rossby waves in shear instability, Phys. Fluids 17, 064107 (2005). 